TRANSACTIONS OF THE

AME RICAN MATHEMATICAL SOCIETY

Volume 169, July 1972

\title{
A SHEAF-THEORETIC DUALITY THEORY FOR CYLINDRIC ALGEBRAS
}

\author{
BY
}

\author{
STEPHEN D. COMER(1)
}

\begin{abstract}
Stone's duality between Boolean algebras and Boolean spaces is extended to a dual equivalence between the category of all andimensional cylindric algebras and a certain category of sheaves of such algebras. The dual spaces of important types of algebras are characterized and applications are given to the study of direct and subdirect decompositions of cylindric algebras.
\end{abstract}

It is a thesis of this paper that certain sheaves serve adequately as the dual spaces of cylindric algebras in the same way that Boolean spaces serve as the dual spaces of Boolean algebras. This duality is described in $\$ 1$. These results are established by algebraically imitating, with suitable cylindric algebra concepts, the sheaf duality theory for rings presented in R. S. Pierce's monograph [6]. These results also hold for other versions of algebraic logic such as polyadic algebras. In $\$ 2$ the dual spaces of locally finite, representable, and regular algebras are characterized; $\$ 4$ gives some applications to the decomposition theory for cylindric algebras.

Our study can be viewed in several ways. In algebraic logic, with each firstorder theory $\Gamma$ there is associated an algebraic structure (called an algebra of formulas) that describes certain aspects of $\Gamma$. Since the theory $\Gamma$ can be determined from the set of all complete theories extending $\Gamma$, the following problem concerning the adequacy of algebraic logic arises. Assuming we know the algebra $\mathfrak{F}_{\Delta}$ associated with each complete (and consistent) theory $\Delta$ extending the theory $\Gamma$, how can we describe the algebra $\mathfrak{F}_{\Gamma}$ associated with $\Gamma$ in terms of all the pairs $\left(\Delta, \mho_{\Delta}\right)$ ? This problem is similar to the one in algebraic geometry of describing the ring associated with an affine variety in terms of the local rings given at each point of the variety. In our situation, if we think of a theory $\Gamma$ as being determined by the set $X_{\Gamma}$ of all complete extensions of $\Gamma$ and think of the algebra of formula $\widetilde{F}_{\Delta}$ as being assigned to each point $\Delta$ of $X_{\Gamma}$, then our problem is of the same nature as the one in algebraic geometry mentioned above. This analogue with algebraic geometry is very close; in $\$ 3$ we solve the logical prob-

Presented to the Society, April 5, 1969 under the title Representation of cylindric algebras by sheaves and January 23, 1970 under the title The dual space of an algebra of formulas; received by the editors October 14, 1970.

AMS 1969 subject classifications. Primary $0240,0242,0248$.

Key words and phrases. Cylindric algebras, sheaves, sectional representations, Boolean spaces, Stone representation the orem, dual space of a cylindric algebra.

(1) Research supported by NSF Grants GP 8725 and GP 11804. 
lem by just imitating the ring theoretic solution to the geometric one. The idea is to consider $X_{\Gamma}$ as a topological space and to glue all of the $\mho_{\Delta}$ 's $\left(\Delta \in X_{\Gamma}\right)$ together with a suitable topology to form a space $\mathcal{S}(\Gamma)$. This is just the construction of a sheaf $\delta(\Gamma)$ of cylindric algebras over the space $X_{\Gamma}$. Then, the algebra $\mathcal{F}_{\Gamma}$ is (up to isomorphism) the algebra $\Gamma\left(X_{\Gamma}, \delta(\Gamma)\right.$ ) of all continuous sections of the sheaf $\left(X_{\Gamma}, \mathcal{S}(\Gamma)\right)$.

The analogue of the problem raised above concerning the adequacy of Boolean algebras for studying propositional theories has a well-known solution. Complete (and consistent) sentential theories extending a given theory $\Gamma$ correspond to maximal ideals in the Boolean algebra $\mathfrak{F}_{\Gamma}$ and the topological Stone representation theorem tells us how to describe $\mathfrak{F}_{\Gamma}$ in terms of these ideals. The representation as all sections of a sheaf is a generalization to $a$-dimensional cylindric algebras of a sheaf-theoretic formulation of Stone's theorem. Not only does the duality between Boolean algebras and Boolean spaces extend but also a form of the correspondence between ideals in Boolean algebras and open subsets in the dual space.

From the viewpoint of universal algebra the representation of an algebra as all sections of a reduced sheaf is a certain subdirect decomposition in which (at least in the nice cases) the factors are directly indecomposable. This representation has the advantage that it is unique in a certain sense. A common universal algebra generalization of this aspect of both the duality results outlined here and the ring representation results in [6] can be found in [1].

0 . Preliminaries. An $\alpha$-dimensional cylindric algebra will be referred to as a $\mathrm{CA}_{\alpha}$ and a Boolean algebra as a BA. Basic facts about $\mathrm{CA}_{\alpha}$ 's may be found in [3] and [4]. In this paper it is convenient to disallow the one element algebra; thus, it is assumed $0 \neq 1$ in all $C A_{\alpha}$ 's. When a class of $C A_{\alpha}$ 's is treated as a category the morphisms are the usual homomorphisms between members of the class.

A central position in this study of a $\mathrm{CA}_{\alpha}$ Q⿱ $\mathrm{V}$ is played by $Z(\mathscr{V})=\{x \in A$ : $\Delta x=0\}$ which forms a Boolean subalgebra of $\mathcal{O}$. $Z(\mathscr{Z})$ plays the same role for $\mathrm{CA}_{\alpha}$ 's that the $\mathrm{BA}$ of central idempotents does for rings in [6]. The connection between cylindric ideals of a $\mathrm{CA}_{a} \mathrm{Ul}$ and $\mathrm{BA}$ ideals of $Z(\mathfrak{U l})$ also plays a major role. For an ideal $J$ of the $B A Z(\mathscr{U}), \bar{J}=\{x \in A: x \leq y$ for some $y \in J\}$ is the smallest $\mathrm{CA}_{\alpha}$ ideal of $\mathcal{A}$ containing $J$; for an ideal $I$ of $\mathscr{U}, I \cap Z(\mathscr{U})$ is a BA ideal of $Z(\mathscr{U})$. Observe that $\bar{J} \cap Z(\mathscr{U})=J$ and $\overline{I \cap Z(\mathfrak{U})} \subseteq I$. An ideal $I$ of $\mathscr{U}$ is called regular if $\overline{I \cap Z(\mathscr{U})}=I$ and a $\mathrm{CA}_{a}$ Q⿱ $\mathrm{U}$ is regular if every ideal of $\mathcal{U}$ is regular. It is known that every $\mathrm{LCA}_{\alpha}$ is regular and it is obvious that every simple $\mathrm{CA}_{a}$ is regular. Regular algebras arose naturally during this investigation; they appear to be a very natural generalization of $\mathrm{LCA}_{\alpha}$ 's and share many of their 
properties. It is easy to show that a $\mathrm{CA}_{\alpha}$ $\mathcal{U}$ is regular if and only if every principal ideal of $2 \mathrm{l}$ is generated by an element of $Z(\mathscr{U})$. This condition is equivalent to: for every $x \in A, \Delta c_{(\Gamma)} x=0$ for some finite subset $\Gamma$ of $\alpha$. For $\alpha<\omega$ every $C A_{\alpha}$ is regular; if $\alpha \geq \omega$ every regular $C A_{\alpha}$ is an $R C A_{\alpha}$ by 2.3 and 2.4 below.

The definition of a sheaf $(X, \mathcal{S}, \pi)$ of $\mathrm{CA}_{a}$ 's and the basic elementary properties of sheaves and sections of sheaves can be obtained from Part I of [6] by replacing the word "ring" by "CA ". In case $\pi$ and/or $X$ are understood, a sheaf of $C A_{\alpha}$ 's is denoted by $(X, \mathcal{S})$ or simply $\mathcal{S}$ and it is called an $\alpha$-space. $\Gamma(X, \mathcal{S})$ denotes the set of all continuous sections of $(X, \mathcal{S}, \pi)$; it is given the structure of a $C A_{\alpha}$ by considering it as a subalgebra of $\Pi_{x \in X} \mathcal{S}_{x}$ where $\mathcal{S}_{x}=$ $\pi^{-1} x$ is the stalk over $x \in X$. If $X$ is a Boolean space, i.e. a totally disconnected, compact Hausdorff space, then $\Gamma(X, \delta)$ is a subdirect product of $\left\{\delta_{x}: x \in X\right\}$. If $X$ is a topological space and a $C A_{\alpha}$ Ut is given the discrete topology, then $\mathcal{S}=X \times A$ with the product topology is a sheaf of $\mathrm{CA}_{\alpha}$ 's over $X$ with each stalk $\mathcal{S}_{x}=\{x\} \times \mathscr{U}$ isomorphic to $\mathscr{U}$. $\mathcal{S}$ is called the trivial $\mathcal{H}$-sheaf over $X$. For this sheaf $\Gamma(X, \delta)$ is isomorphic to the $C A_{\alpha}$ of all continuous functions from $X$ into 2 .

1. Duality theory. Contravariant functors between the category of all $\mathrm{CA}_{a}{ }^{\prime} \mathrm{s}$ and a certain category of $\alpha$-spaces are described below. The basic properties of these functors and the duality they determine are established by replacing ring notions by suitable $\mathrm{CA}_{\alpha}$ notions in the arguments given in Part I of [6].

An $\alpha$-space $(X, \delta)$ is called reduced if $X$ is a Boolean space and, for all $\sigma \in Z(\Gamma(X, \delta))$ and $x \in X$, either $\sigma(x)=0_{x}$ or $\sigma(x)=1_{x}$. The following proposition is useful for identifying $\alpha$-spaces as reduced. The condition $(*)$ is not, in general, necessary (cf. Example 2.5).

Proposition 1.1. If $(X, \mathfrak{S})$ is an a-space where $X$ is a Boolean space then $(X, \mathcal{S})$ is reduced if

(*) $\mathcal{S}_{x}$ is directly indecomposable for all $x \in X$.

If $(X, \delta)$ is a regular $C A_{\alpha}$ (in particular an $\left.L C A_{\alpha}\right)$, then $(*)$ is also necessary.

Observe that a trivial $\mathcal{U}$-sheaf $(X, \mathcal{S})$ is reduced if and only if $X$ is a Boolean space and $\mathscr{A}$ is directly indecomposable. Unlike the situation for rings, a subsheaf of a reduced sheaf of $\mathrm{CA}_{a}$ 's is always reduced.

We now describe a functor that associates to each $C A_{\alpha}$ Ul a reduced $\alpha$-space $(X(\mathscr{U}), \mathcal{S}(\mathscr{U}))=\mathscr{U}^{d}$ called the dual space of $\mathscr{U}$. The base space $X(\mathscr{U})$ is the usual Boolean dual space of the BA $Z(\mathscr{U})$. To be concrete take $X(\mathscr{U})$ as the set of all maximal ideals of $Z(\mathscr{U})$; this becomes a Boolean space when we take the 
collection of all sets $N(y)=\{M \in X(\mathscr{U}): y \notin M\}$ as a basis for the topology. For $M \in X(\mathfrak{U})$, let $\mathcal{S}_{M}(\mathfrak{U})=\mathscr{U} / \bar{M}$ (the stalk over $M$ ) and $\mathcal{S}(A)=\bigcup\left\{\mathcal{S}_{M}(\mathscr{Q}): M \in X(\mathfrak{U})\right\}$. Note that $\mathcal{S}_{M_{1}}(\mathscr{U})$ and $\mathcal{S}_{M_{2}}(\mathscr{Q})$ are disjoint when $M_{1} \neq M_{2}$. The projection $\pi: \quad \mathcal{( Q U}) \rightarrow X(\mathscr{U})$ is defined for $s \in \mathcal{S}_{M}(\mathscr{U})$ by $\pi(s)=M$. To describe the topology on $\mathcal{S}(\mathscr{L})$ we need some auxiliary functions. For $a \in A$ we define a function $\sigma_{a}$ : $X(\mathscr{U}) \rightarrow \mathcal{S}(\mathscr{U})$ by $\sigma_{a}(M)=a / \bar{M}$ for all $M \in X(\mathscr{U})$. The topology given to $\mathcal{S}(\mathscr{U})$ is the smallest topology for which all $\sigma_{a}$ 's $(a \in A)$ are open.

It turns out that $\mathscr{U}^{d}=(X(\mathscr{U}), \mathcal{S}(\mathscr{U}))$ is a reduced $\alpha$-space and that the mapping $\xi_{\mathfrak{q}}: \mathscr{U} \rightarrow \Gamma(X(\mathscr{U}), \mathcal{S}(\mathscr{U}))$ defined by $\xi_{\mathfrak{q}}(a)=\sigma_{a}$ is an isomorphism. In particular, under $\xi_{\mathscr{q}}$, an element $a \in Z(\mathcal{U})$ corresponds with the characteristic function $\sigma_{N} \in \Gamma(X(\mathscr{U}), \mathcal{S}(\mathscr{U}))$ of the clopen subset $N=N(a)$ of $X(\mathscr{U})$.

To make the correspondence $\mathscr{U} \omega \mathcal{U}^{d}$ into a functor we describe the dual $\lambda^{d}=\left(\bar{\lambda}, \lambda^{0}\right)$ of a homomorphism $\lambda: \mathscr{U} \rightarrow \mathscr{B}$. For $M \in X(\mathbb{B})$ let $\bar{\lambda}(M)=$ $\lambda^{-1}(M) \cap Z(\mathscr{U}) ; \bar{\lambda}$ is a continuous map of $X(\mathscr{B})$ into $X(\mathscr{U})$. For $M \in X(\mathscr{B})$ and $a \in A$ define $\lambda^{0}(M, a / \overline{\bar{\lambda}} M)=\lambda(a) / \bar{M}$. Then $\lambda^{0}(M, \cdot)$ is a homomorphism of $\widehat{S}_{\bar{\lambda} M}(\mathscr{l})$ into $\mathcal{S}_{M}(\mathscr{B})$ and $\lambda^{d}$ is a sheaf morphism of $\mathscr{B}^{d}$ into $\mathfrak{U}^{d}$.

The functor from the category of all reduced $\alpha$-spaces (and sheaf morphisms) to the category of $\mathrm{CA}_{a}$ 's is easier to describe. For an $\alpha$-space $\mathcal{O}=(X, \delta)$ let $\mathcal{O}^{*}=\Gamma(X, \mathcal{S})$; if $\Lambda=(\lambda, \phi)$ is a sheaf morphism from the a-space $(X, \mathcal{S})$ into $(Y, \mathscr{R})$, the dual $\Lambda^{*}: \Gamma(Y, \mathcal{R}) \rightarrow \Gamma(X, \delta)$ of $\Lambda$ is defined by requiring $\left(\Lambda^{*} \sigma\right)(x)=\phi(x, \sigma(\lambda x))$ for $x \in X$ and $\sigma \in \Gamma(Y, R)$.

The following theorem justifies calling $\mathscr{Q}^{d}$ the dual space of $\mathcal{U}$. (Cf. Theorem 6.6 in [6].)

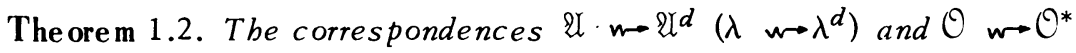
$\left(\Lambda \mathrm{\omega} \rightarrow \Lambda^{*}\right)$ are contravariant functors between the category of all $C A_{\alpha}{ }^{\prime}$ s and the category of all reduced a-spaces. Further, there exist natural isomorphisms

$$
\xi_{\mathscr{r}}: \quad \mathfrak{Q} \cong\left(\mathscr{U}^{d}\right)^{*}, \quad \eta_{\mathcal{O}}: \mathcal{O} \cong\left(\mathcal{O}^{*}\right)^{d}
$$

showing that the categories are dual equivalent.

The isomorphism $\xi_{\mathfrak{r}}$ in 1.2 is the function defined above.

The preceding theorem raises the problem of finding the dual of various cylindric notions. We mention a few. For a $C A_{a}$ homomorphism $\lambda$ : $\mathfrak{A} \rightarrow \mathfrak{B}$ we always have $\lambda(Z(\mathscr{U})) \subseteq Z(B)$. For $\lambda$ onto $\mathscr{E}$ we say that $\lambda$ is a conformal epi if $\lambda(Z(\mathscr{U}))=Z(\mathfrak{B})$. A regular $C A_{\alpha} \mathfrak{A}$ (in particular an $\mathrm{LCA}_{\alpha}$ ) has the property that for any $B$ and $\lambda$ mapping $\mathscr{U}$ onto $B, \lambda$ is a conformal epi. An example of a $C A_{\alpha}$ without this property is given in 2.5. The dual of the notion of conformal epi is very nice-it is a sheaf morphism $(\lambda, \phi)$ where $\lambda$ is one-to-one and $\phi$ restricted to each stalk is onto. The proof is similar to the one given for rings [6]. 
Also as with rings we can extend to $\mathrm{CA}_{a}$ 's a form of the correspondence between ideals of BA's and open subsets of Boolean spaces. Let $\operatorname{Id}^{R}(\mathscr{U})$ denote the set of all regular ideals of $\mathfrak{U} . \mathrm{Id}^{R}(\mathfrak{U})$ is a sublattice of the lattice Id( $\left.\mathscr{U}\right)$ of all ideals of $\mathscr{U}$. For $\sigma \in \Gamma(X, \mathcal{S})$ we call the closed subset $\|\sigma\|=\{x \in X$ : $\left.\sigma(x) \neq 0_{x}\right\}$ the support of $\sigma$. For any subset $U$ of $X$ define $J[U]=\{\sigma \epsilon$ $\Gamma(X, \delta):\|\sigma\| \subseteq U\}$ and for any subset $J$ of $\Gamma(X, \mathcal{S})$ define $U[J]=$ $\mathcal{U}\|\| \sigma: \sigma \in J\}$. The following is proved in the same way as the corresponding theorem for rings (see 9.3 of [6]).

Theorem 1.3. The function $J \mathrm{~m} \rightarrow U[J]$ is an isomorphism from $\operatorname{Id}^{R}(\Gamma(X, \mathfrak{S}))$ onto the lattice of all open subsets of $X$; its inverse is the function mapping $U$ to $J[U]$.

Using 1.3 the problem of characterizing the class of ideal lattices of certain $\mathrm{CA}_{a}$ 's can be reduced to the same problem for BA's. The latter problem is fairly easy.

Corollary 1.4. If $K$ denotes the class of all BA's, $L C A_{\alpha}$ 's, regular $C A_{\alpha}$ 's, or (if $\alpha<\omega) C A_{\alpha}$ 's and $L$ is a lattice, then the following are equivalent.

(i) $L$ is a complete, compactly generated pseudo BA in which an element is compact if and only if it is complemented;

(ii) $L$ is isomorpbic to $\operatorname{Id}(\mathfrak{U})$ for some $\mathscr{U}$ in $K$;

(iii) $L$ is isomorpbic to $\mathrm{Id}^{R}(\mathfrak{U})$ for some $C A_{\alpha}$ UU.

Parts of 1.4 are mentioned in [3]; (i) does not characterize the class of ideal lattices of $\mathrm{CA}_{a}$ 's when $\alpha \geq \omega$.

2. Duals of LCA Ind $_{\alpha}$ RCA ${ }_{\alpha}$ 's. In this section we are interested in describing the dual spaces of $\mathrm{LCA}_{\alpha}$ 's, $\mathrm{RCA}_{\alpha}$ 's and regular $\mathrm{CA}_{\alpha}$ 's. If $\mathcal{U}$ is an $\mathrm{LCA}_{\alpha}$ and $(X, \mathfrak{S})$ its dual space, then $\mathfrak{U} \cong \Gamma(X, \mathfrak{S})$, so 1.1 implies each stalk $\mathcal{S}_{x}$ is directly indecomposable and hence simple since $\mathcal{S}_{x}$ is an LCA ${ }_{a}$. This suggests that we look for the duals of $\mathrm{LCA}_{\alpha}$ among the following type of $\alpha$-spaces.

We say an $a$-space $(X, \mathcal{S})$ is regular if $X$ is a Boolean space and $\mathcal{S}$ is a sheaf of simple $C A_{\alpha}$ 's (i.e. $\mathcal{S}_{x}$ is simple for all $x \in X$ ). By 1.1 , a regular $\alpha$-space is reduced.

Before characterizing the dual space of an $\mathrm{LCA}_{\alpha}$ we need to observe an additional property.

Lemma 2.1. If $(X, \mathfrak{S})$ is the dual space of an $L C A_{\alpha} \mathfrak{U}$, then for every $s \in \mathcal{S}$ there is a neighborbood $T$ of $s$ such that $\Delta t \subseteq \Delta s$ for every $t \in T$.

Proof. It is enough to show that if $\sigma_{x} \in \Gamma(X(\mathfrak{U}), \mathcal{S}(A))$ and $M \in X(\mathfrak{U})$, there is a neighborhood $\Re$ of $M$ for which $\Delta \sigma_{x}(N) \subseteq \Delta \sigma_{x}(M)$ for all $N \in \Re$. Since 
$\Delta x$ is finite, $z=\Sigma\left\{c_{\kappa} x \oplus x: \kappa \in \Delta x \sim \Delta \sigma_{x}(M)\right\}$ is in $\mathcal{U}$. Thus, $N\left(-c_{(\Delta z)} z\right)$ is the desired neighborhood of $M$.

Let us call an $\alpha$-space $(X, \delta)$ locally finite if (i) each stalk is an LCA , and (ii) every $s \in \mathcal{S}$ has a neighborhood $T$ in which $\Delta t \subseteq \Delta s$ for all $t \in T$. Under assumption (i) it can be shown that (ii) is equivalent to the apparently stronger condition that every $s \in \mathcal{S}$ has a neighborhood in which every element has the same dimension set as $s$.

Theorem 2.2. An a-space $(X, \mathcal{S})$ is the dual space of an $L C A_{\alpha}$ if and only if $(X, \mathfrak{S})$ is a locally finite regular a-space. Consequently the functors in 1.2 give a duality between $L C A_{a}$ 's and locally finite regular a-spaces.

Proof. In view of 1.1 and 2.1 , it suffices to show that $\Gamma(X, \mathcal{J})$ is an $\mathrm{LCA}_{\alpha}$ whenever $(X, \mathcal{S})$ is a regular, locally finite $\alpha$-space. Suppose we have such a $(X, \mathfrak{S})$ and $\sigma \in \Gamma(X, \mathfrak{S})$. It follows that for each $x \in X$ there is a neighborhood $N_{x}$ of $x$ such that $\Delta \sigma(y) \subseteq \Delta \sigma(x)$ for all $y \in N_{x}$. Applying the partition property (see [6, p. 12]), there exist a finite number of clopen sets $N_{i}$ for $i<n$ which partition $X$ and such that, for each $i<n, N_{i} \subseteq N_{x_{i}}$ for some $x_{i} \in X$. Now $\Delta \sigma \subseteq$ $\mathcal{U}\left\{\Delta \sigma\left(x_{i}\right): i<n\right\}$ which is finite. It follows that $\Gamma(X, \mathfrak{S})$ is an LCA . .

Another important class of $\mathrm{CA}_{\alpha}{ }^{\prime}$ 's is the class $\mathrm{RCA}_{\alpha}$ of all representable $\mathrm{CA}_{\alpha}$ 's. It is well known that this is an equational class, so, on the strength of general facts about sheaves over Boolean spaces, the following holds.

Theorem 2.3. $(X, \mathcal{S})$ is the dual space of an $R C A_{\alpha}$ if and only if it is a reduced a-space of $R C A_{\alpha}$ 's. Consequently, the functors in 1.2 give a duality between $R C A_{\alpha}$ 's and reduced a-spaces of $R C A_{\alpha}$ 's.

For $\alpha<\omega$ every $\mathrm{CA}_{\alpha}$ is locally finite; so every reduced $\alpha$-space is regular. This is not true for $\alpha \geq \omega$. It is known from [3] that, for $\alpha \geq \omega$, simple CA 's are representable; thus, the class of regular $\alpha$-spaces lie between the class of regular locally finite $\alpha$-spaces and the class of reduced $\alpha$-spaces of RCA 's. By the following result regular $\mathrm{CA}_{\alpha}$ 's are the duals of regular $\alpha$-spaces and consequently are representable if $\alpha \geq \omega$.

Theorem 2.4. $(X, \delta)$ is the dual of a regular $C A_{\alpha}$ if and only if it is a regular a-space. Consequently 1.2 gives a duality between regular $C A_{\alpha}$ 's and regular $\alpha$-spaces.

The proof of 2.4 is similar to that of 1.11 in [6] using in the appropriate places the property of simple CA ${ }_{a}$ 's $\mathcal{S}_{x}$ that for every $0 \neq a \in \mathcal{S}_{x}, c_{(F)} a=1$ for some finite subset $F$ of $\alpha$. From the proof of 2.4 it follows that $\|\sigma\|$ is clopen whenever $\sigma \in \Gamma(X, \mathfrak{S})$ where $(X, \delta)$ is regular. Consequently, when $(X, \delta)$ is a 
regular $\alpha$-space (in particular, the dual of an $\mathrm{LCA}_{\alpha}$ ) the topology on $\mathfrak{S}$ is Hausdorff.

For $a \geq \omega$, there are simple $\mathrm{CA}_{\alpha}$ 's which are not $\mathrm{LCA}_{\alpha}$ 's (see [3]); hence, the class of regular $\mathrm{CA}_{\alpha}$ 's properly includes the class of $\mathrm{LCA}_{\alpha}$ 's. A regular $C A_{a}$ is clearly semisimple. The example below (with (S) simple) shows that a semisimple $C A_{a}$ does not have to be regular and, moreover, the stalks of the dual space of a semisimple $\mathrm{CA}_{a}$ do not even need to be directly indecomposable. (Cf. 1.1 and remark on conformal epi's following 1.2.)

Example 2.5. Let (S be a directly indecomposable $\mathrm{CA}_{\alpha}, \alpha \geq \omega$, and let $I$ be the set of all finite subsets of $\alpha$. Choose an ultrafilter $F$ on $I$ such that $\{\Delta \in I: \Delta \supseteq \Gamma\} \in F$ for all $\Gamma \in I$. We claim

(1) The epimorphism $\lambda:{ }^{I}(5){ }^{I}(S / F$ induced by the ultrafilter $F$ is not conformal. (Consequently, ${ }^{I}(S)$ is not regular.)

We must construct an element with dimension set 0 in the ultrapower ${ }^{I}(S / F$ that is not the image of an element in $Z{ }^{I}(5)$. Choose a one-to-one $\sigma: I \rightarrow I$ such that, for all $\Gamma \in I, \Gamma \cap \sigma(\Gamma)=0$ and $\sigma(\Gamma)$ contains at least two elements. Define $f \in \epsilon^{I}(5)$ by $f(\Gamma)=d_{\sigma(\Gamma)}=\Pi\left\{d_{\kappa \lambda}: \kappa, \lambda \in \sigma(\Gamma)\right\}$ for $\Gamma \in I$. Since, for each $\kappa<\alpha,\{\Gamma \in I: \quad \Gamma \supseteq\{\kappa\}\} \subseteq\left\{\Gamma \in I: \quad c_{\kappa} f(\Gamma)=f(\Gamma)\right\} \in F, \Delta(f / F)=0$.

Also observe that $f / F \neq 0 / F$ and $f / F \neq 1 / F$. This is true since $f(\Gamma)=d_{\sigma(\Gamma)} \neq 0^{\sigma}$ and $f(\Gamma) \neq 1^{\mathbb{E}}$ for all $\Gamma \in I$. To show the $\lambda$ in (1) is not conformal it is enough to show $f / F \notin \lambda\left(Z\left(^{I}(\mathfrak{S})\right)\right.$. For $g \in Z\left(^{I}(\mathbb{S}), \Delta g=\bigcup\{\Delta g(\Gamma): \Gamma \in I\}=0\right.$ and $Z(\mathbb{S})=$ $\{0,1\}$ so $\{\Gamma \in I: g(\Gamma)=0\} \cup\{\Gamma \in I: g(\Gamma)=1\}=I \in F$. Thus, one of the two sets above belongs to $F$ implying that $g / F=0 / F$ or $g / F=1 / F$. Consequently, $f / F \notin\left(Z\left({ }^{I}(\mathbb{S})\right)\right.$ and (1) holds.

Implicit in the above proof of (1) is a description of the dual space of a product of directly indecomposable $\mathrm{CA}_{\alpha}$ 's. We state the general result. Then

Proposition 2.6. Suppose $\mathscr{U}=\Pi_{i \in I} B_{i}, B_{i}$ directly indecomposable $C A_{a}$ 's.

(a) $Z(\mathscr{U})={ }^{I}\{0,1\}$ and $X(\mathscr{U})$ is the Stone space of ${ }^{\prime} 2$.

(b) Since there is a biunique correspondence between the maximal ideals of $Z(\mathscr{U})$ and the ultrafilters on $I$, the stalk $\mathcal{S}_{M}(\mathscr{U})$ of $\mathfrak{Q}^{d}$ over $M \in X(\mathscr{U})$ is just the ultraproduct $\Pi_{i} \in I B_{i} / F$ where $F$ is the ultrafilter on $I$ corresponding to $M$.

3. Sheaves and theories. In this section we give an interpretation of the dual space of the algebra of formulas associated with a standard first order theory. It is this interpretation that yields a solution to the question posed in the introduction. The interpretation can obviously be extended to theories in other languages; however, we restrict ourselves to a standard first order language $L$ with equality in which we have variables $v_{i}$ for $i<\omega$ and each predicate symbol has finite rank. The set $F m l_{L}$ of formulas is defined by recursion in 
the usual way. Sentences are formulas without free variables; the set of $L$-sentences is denoted by $S_{e n t}$. A set $\Gamma$ of $L$-sentences is a theory if for every $\phi \in$ Sent $_{L}, \Gamma \vdash \phi$ implies $\phi \in \Gamma$. A theory $\Gamma$ is complete if $\Gamma$ is consistent and either $\phi \in \Gamma$ or $\neg \phi \in \Gamma$ for every $L$-sentence $\phi$. For information on the relationship between languages and $\mathrm{CA}_{a}$ 's see [4].

An LCA $\omega \mathfrak{F}_{\Gamma}^{L}$, called the algebra of formulas of $\Gamma$, can be associated with an $L$-theory $\Gamma$. For an $L$-theory $\Gamma$ define

$$
\Xi_{\Gamma}^{L}=\left\{(\phi, \psi): \phi, \psi \in \text { Fmla }_{L}, \Gamma \vdash \phi \leftrightarrow \psi\right\} .
$$

The elements of $\mathcal{F}_{\Gamma}^{L}$ are $\Xi_{\Gamma}^{L}$-equivalence classes of $L$-formulas; the cylindric operations on $i_{F}^{L}$ are the natural quotient operations induced on $\Xi_{\Gamma}^{L}$-equivalence classes by the analogous logical operations on $F m l a_{L}$. For brevity, we denote the $\Xi_{\Gamma}^{L}$-class of a formula $\phi$ by $[\phi]_{\Gamma}$.

It is easily seen that $\mho_{\Gamma}^{L}$ is an LCA ${ }_{\omega}$ and that $Z\left(\tau_{\Gamma}^{L}{ }_{\Gamma}^{L}\right)=\operatorname{Sent}_{L} / \Xi_{\Gamma}^{L}$. For an $L$-theory $\Gamma$ let $X_{\Gamma}$ be the set of all complete theories of $L$ extending $\Gamma$. For $\Delta \in X_{\Gamma}$ let $M[\Delta]=\left\{\left[\neg_{\phi}\right]_{\Gamma}: \phi \in \Delta\right\}$ and for $M \in X\left(\mathcal{F}_{\Gamma}^{L}\right)$ let $\Delta[M]=\left\{\phi \in\right.$ Sent $_{L}$ : $\left.[\neg \phi]_{\Gamma} \in M\right\}$. For a theory $\Gamma$, define $\mathcal{S}(\Gamma)=\bigcup_{\Delta \in X_{\Gamma}}\{\Delta\} \times \mathfrak{F}_{\Delta}^{L}$ (the disjoint union of $\left\{\left\{_{\Delta}^{L}: \Delta \in X_{\Gamma}\right\}\right.$ ).

Theorem 3.1. There exist suitable topologies on $X_{\Gamma}$ and $\mathcal{S}(\Gamma)$ such that $\left(X_{\Gamma}, \mathfrak{S}(\Gamma)\right)$ is (up to sheaf is omorphism) the dual space of $\mathfrak{F}_{\Gamma}^{L}$.

Proof. It is well known (see [4]) that the correspondences $\Delta m \rightarrow M[\Delta]$ and $M \leftrightarrow \Delta[M]$ are inverse one-to-one functions between $X_{\Gamma}$ and $X\left(\mathfrak{F}_{\Gamma}^{L}\right)$. Using these functions $X_{\Gamma}$ can be made into a Boolean space. The relation $f=$ $\left.\left\{(\phi]_{\Gamma},[\phi]_{\Delta}\right): \phi \in F m l a_{L}\right\}$ is a homomorphism of $\mathfrak{F}_{\Gamma}^{L}$ onto $\mathfrak{F}_{\Delta}^{L}$. The ideal $I$ of $\mathfrak{F}_{\Gamma}^{L}$ associated with $f$ is $I=\left\{[\phi]_{\Gamma}: \Delta \vdash \neg \phi\right\}$; thus, $I \cap Z\left(\mathfrak{F}_{\Gamma}^{L}\right)=M[\Delta]$. Since LCA $\omega^{\prime}$ 's are regular, $I=\overline{M[\Delta]}$ and $\xi_{\Delta}^{L} \cong \mathcal{F}_{\Gamma}^{L} / \overline{M[\Delta]}$. The disjoint union of the above isomorphisms gives a one-to-one function $b$ from $\delta\left(\mathcal{F}_{\Gamma}^{L}\right)$ onto $\delta(\Gamma)$; transferring the topology to make $b$ a homeomorphism, $\left(X_{\Gamma}, \mathcal{S}(\Gamma)\right)$ becomes an a-space and the maps $\Delta \rightsquigarrow M[\Delta]$ and $b$ give rise to a sheaf isomorphism of $\left(X_{\Gamma}, \mathcal{S}(\Gamma)\right)$ onto $\left(X\left(\mathcal{F}_{\Gamma}^{L}\right), \mathcal{S}\left(\mathfrak{F}_{\Gamma}^{L}\right)\right)$.

For completeness we describe the topologies on $X_{\Gamma}$ and $\mathcal{S}(\Gamma)$ arising in the above proof. Note that these topologies are quite natural and do not depend on the duality theory. A basis for the topology on $X_{\Gamma}$ is the collection of all sets $N(\phi)=\left\{\Delta \in X_{\Gamma}: \phi \in \Delta\right\}$ for $\phi \in$ Sent $_{L}$. A basis for the desired topology on $\mathcal{S}(\Gamma)$ is the collection of all sets $B_{\phi, \psi}=\left\{\left(\Delta,[\phi]_{\Delta}\right): \psi \in \Delta, \Delta \in X_{\Gamma}\right\}$ where $\phi \in$ Fmla $_{L}$ and $\psi \in$ Sent $_{L}$.

The duality result 1.2 and 3.1 allows us to determine $\mho_{\Gamma}^{L}$ in terms of $\left\{\mathfrak{F}_{\Gamma}^{L}: \Delta \in X_{\Gamma}\right\}$ solving the question posed in the introduction. 
Corollary 3.2. For an L-theory $\Gamma, \mathfrak{F}_{\Gamma}^{L} \cong \Gamma\left(X_{\Gamma}, \mathcal{S}(\Gamma)\right)$.

If $\mathcal{S}$ is an $\alpha$-space over $X$ and $Y \subseteq X$, then $\mathcal{S} \mid Y=\pi^{-1}(Y)$ is a sheaf over $Y$ called the restriction of $(X, \delta)$ to $Y$. If $(X, \delta)$ is a reduced $a$-space, $Y$ a closed subset of $X$, and every $s \in \mathcal{S}$ has a neighborhood $T$ such that $\Delta t \subseteq \Delta s$ for all $t \in T$, then a function extension argument shows that $(Y, \mathcal{S} \mid Y)$ is a reduced $\alpha$-space. If $(X, \mathcal{S})$ is a regular $\alpha$-space and $Y$ is a closed subset of $X$ then $(Y, \mathcal{S} \mid Y)$ is regular. In particular, either of the two statements above can be used to show that the restrictions of a locally finite regular $\alpha$-space to a closed subset gives a locally finite regular $\alpha$-space. The following result shows that a restriction of $\left(\mathfrak{F}_{\Gamma}^{L}\right)^{d}$ is the dual space of another $L$-theory. Denote by $\Omega$ the $L$-theory consisting of all logically valid $L$-sentences.

Proposition 3.3. For a language $L$, the dual spaces of algebras of formulas associated with L-theories correspond to restrictions of $\left(X_{\Omega}, \mathcal{S}(\Omega)\right)$ to closed subsets of $X_{\Omega}$.

Proof. It is well known that for any $L$-theory $\Gamma$ there is a conformal epi $\lambda$ : $\mathfrak{F}_{\Omega}^{L} \rightarrow \mathfrak{F}_{\Gamma}^{L}$ given by $\lambda\left([\phi]_{\Omega}\right)=[\phi]_{\Gamma}$ for every formula $\phi$. The dual morphism $\lambda^{d}=\left(\bar{\lambda}, \lambda^{0}\right):\left(X_{\Gamma}, \mathcal{S}(\Gamma)\right) \rightarrow\left(X_{\Omega}, \mathcal{S}(\Omega)\right)$ is easy to describe: for $\Delta \in X_{\Gamma}$ and $\left(\Delta,[\phi]_{\Delta}\right) \in \mathcal{S}(\Omega)_{\Delta}, \bar{\lambda}(\Delta)=\Delta$ and $\lambda^{0}\left(\Delta,\left(\Delta,[\phi]_{\Delta}\right)\right)=\left(\Delta,[\phi]_{\Delta}\right) \in \mathcal{S}(\Gamma)_{\Delta}$. Thus, $\lambda^{d}$ is an isomorphism of $\left(X_{\Gamma}, \mathcal{S}(\Gamma)\right)$ onto the restriction of $\left(X_{\Omega}, \mathcal{S}(\Omega)\right)$ to the closed subset $X_{\Gamma}$ of $X_{\Omega}$. Moreover, every restriction of $\left(X_{\Omega}, \mathcal{S}(\Omega)\right)$ to a closed subset $Y$ of $X_{\Omega}$ is (up to isomorphism) the dual space of an algebra of formulas; for if $\Gamma=\bigcap Y$, then $Y=X_{\Gamma}$ since $Y$ is closed and the dual space of $\mho_{\Gamma}^{L}$ is isomorphic to $(Y, \mathcal{S}(\Omega) \mid Y)$.

4. Applications to the decomposition theory of $\mathrm{CA}_{\alpha}$ 's. In this section we give some easy applications of sheaf theory to the study of decompositions of $\mathrm{CA}_{\alpha}$ 's. Trivial sheaves will be used to construct direct and subdirect decompositions with specific properties. An extensive study of the properties of direct and subdirect decompositions of $\mathrm{CA}_{\alpha}$ 's can be found in [3]. Several of the results to follow are new; for those which are known, the proofs and/or viewpoint is different from [3].

In [3] it is proved that every $\mathrm{CA}_{\alpha}$ has the refinement property. Consequently, a direct decomposition of a $\mathrm{CA}_{\alpha}$ into directly indecomposable factors is unique (up to isomorphism). In [2] Hanf proved that various pathological direct decompositions can exist for BA's. Theorem 4.2 shows that these decompositions can exist for nondiscrete $\mathrm{CA}_{\alpha}$ 's ( $\mathrm{LCA}_{\alpha}$ 's) as well. The following lemma is essentially due to Jónsson [5]; its proof (in a nonsheaf theoretic form) and 4.2 can be found in [3]. We include it here because it is a nice application of the trivial sheaf construction. In the following $B \mid a$ denotes the relativized $\mathrm{BA}$ and 
2U $f a$ denotes the relativized $\mathrm{CA}_{\alpha}$.

Lemma 4.1. If $B$ is $a B A$ and $(5)$ is a directly indecomposable $C A_{\alpha}$, there is a $C A_{\alpha}$ OU and an isomorphism $f: B \rightarrow Z(\mathscr{U})$ such that the following bold:

(i) for all $a, b \in B, \mathfrak{B}|a \cong \mathfrak{B}| b$ iff Ul $|a \cong \cong \mathfrak{U}| f b$;

(ii) if (5) is an $L C A_{a}$, so is थ;

(iii) for any variety $V$ of $C A_{a}$ 's, $\mathcal{Q} \in V$ iff $(\mathbb{S} \in V$.

Proof. Given $B$ and $(S$ as above let $X$ be the Stone space of $B$ and $(X, X \times(\mathbb{S})$ the trivial $\mathbb{S}$-sheaf over $X$. Then $\mathcal{Q}=\Gamma(X, X \times(\mathfrak{S})$ is the desired algebra. The function $f$ is defined by $f(b)=\sigma_{N(b)}$ (the characteristic function of $N(b))$ for $b \in B$. It follows from $\$ 1$ that $f$ is an isomorphism. Parts (ii) and (iii) are obvious from the properties of sheaves so it is enough to verify (i). If श्र $|f a \cong \mathbb{2}| f b$, then $Z(\mathfrak{Q} \mid f a) \cong Z(\mathfrak{U} \mid f b)$; thus $B|a \cong B| b$ follows. Now suppose $\mathfrak{B}|a \cong \mathbb{B}| b$. Then there is a homeomorphism mapping $N(a)$ one-to-one onto $N(b)$; this homeomorphism induces an isomorphism of the trivial $(5$-sheaf $(N(a)$, $N(a) \times(\mathfrak{S})$ over $N(a)$ onto the trivial (5-sheaf $(N(b), N(b) \times(\mathfrak{S})$ over $N(b)$. Since $N(a)$ is a clopen subset of $X$, the restriction mapping gives an isomorphism थ्य $\mid f a \cong \Gamma(N(a), N(a) \times$ (5). Similarly, $20 \mid f b \cong \Gamma(N(b), N(b) \times($ ( $)$. It follows that थU| $f a \cong$ थी $f b$ as desired.

As a consequence of 4.1 and Hanf's result [2] the following result is immediate.

Theorem 4.2. For any a there are nondiscrete $L C A_{\alpha}$ 's 2 , $B$, $\mathbb{S}$ such that

(i) $\mathfrak{U} \cong \mathscr{U} \times \mathbb{B} \times \mathfrak{B}$ and $\mathfrak{U} \not \mathfrak{U} \times \mathfrak{B}$;

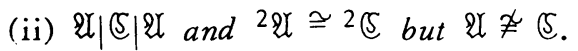

Moreover, a nondiscrete $C A_{a}$ थी may be chosen to belong to any given variety and not to another.

The various other pathological kinds of decompositions established by Hanf can also be extended to nondiscrete $\mathrm{CA}_{\alpha}$ 's. For other uses of 4.1 see [3].

Next we turn to the study of subdirect decompositions. We restrict ourselves to the following unique decomposition properties.

Definition 4.3. (i) $\mathrm{A} \mathrm{CA}_{a}$ थl has the strict unique irredundant subdirect decomposition property if whenever $\left\langle K_{i}: i \in I\right\rangle$ and $\left\langle L_{j}: j \in J\right\rangle$ are two systems of ideals of 2 such that

(1) $\bigcap_{i \in I} K_{i}=\{0\}=\bigcap_{j \in J} L_{j}$;

(2) $\mathscr{U} / K_{i}$ and $U / L_{j}$ are subdirectly indecomposable for $i \in I, j \in J$;

(3) $K_{i} \nsubseteq K_{i}$, and $L_{k} \nsubseteq L_{j}$, for $i, i^{\prime} \in I, j, j^{\prime} \in J$ with $i \neq i^{\prime}$ and $j \neq j^{\prime}$; then $|I|=|J|$ and there is a one-to-one function $f$ of $I$ onto $J$ such that $K_{i}=$ $L_{f(i)}$ for all $i \in I$. 
(ii) $\mathrm{A} \mathrm{CA}_{a}$ थी has the unique irredundant subdirect decomposition property if whenever $\mathscr{U} \cong \mid \subseteq \subseteq_{s d} \Pi_{i \in I} B_{i}$ and $\mathscr{U} \cong \mid \subseteq_{s d} \Pi_{j \in J} \mathfrak{S}_{j}$ with $\mathfrak{B}_{i}$ and $\mathfrak{S}_{i}$ subdirectly indecomposable such that for no $I^{\prime} \subset I$ and no $J^{\prime} \subset J$ is $\mathscr{U} \cong \mid \subseteq_{s d} \Pi_{i \in I}, B_{i}$ and $\mathcal{Q} \cong \mid \subseteq_{s d} \Pi_{j \in J}, \mathbb{S}_{j}$, then $|I|=|J|$ and there is a one-to-one function $f$ of $I$ onto $J$ such that $\mathscr{B}_{i} \cong \mathbb{S}_{f(i)}$ for all $i \in I$.

It is easily seen, on general algebraic grounds, that 4.3 (i) implies 4.3 (ii) and that 4.3 (i) is implied by the strict refinement property. In [3] only 4.3 (ii) is considered. It is shown in 2.4 .42 that the unique subdirect decomposition property 4.3 (ii) holds for a $\mathrm{CA}_{\alpha}$ when only finitely many factors are involved (i.e. 4.3 (ii) with the additional assumption that $|I|,|J|<\omega$ ). The proof of 2.4.42 actually establishes a stronger result. Namely, it is shown that any two finite systems of ideals in a $\mathrm{CA}_{\alpha}$ have a strict refinement. The general algebraic implications mentioned above then establish 4.3 (ii) in case $|I|,|J|<\omega$. In 4.4 we show that 4.3 (ii) holds with restrictions for a reasonably large class of $C A_{\alpha}$ 's including all LCA ${ }_{\alpha}$ 's. We then give a few examples to show that 43(i) fails very often even for $\mathrm{LCA}_{\alpha}$ 's.

Proposition 4.4. If 2 is a regular $C A_{a}$, then 2 thas the unique subdirect decomposition property 4.3 (ii).

Proof. Suppose $\left\langle\mathbb{B}_{i}: i \in I\right\rangle$ and $\left\langle\mathbb{E}_{j}: j \in I\right\rangle$ are two systems of subdirectly indecomposable $\mathrm{CA}_{\alpha}$ 's each giving a subdirect decomposition of $\mathcal{Q}$ as in the hypothesis of 4.3 (ii). Thus, there exist two systems of ideals $\left\langle K_{i}: i \in l\right\rangle$ and $\left\langle L_{j}: j \in J\right\rangle$ of $\mathcal{U}$ satisfying conditions (1)-(3) in $4.3(i)$ such that $\mathscr{U} / K_{i} \cong \mathbb{B}_{i}$ and $\mathscr{U} / L_{j} \cong \mathbb{S}_{j}$. For $i \in I$ and $j \in J$ let $M_{i}=K_{i} \cap Z(\mathscr{U})$ and $N_{j}=L_{j} \cap Z(\mathscr{U})$. Conditions (1)-(3) or 4.3 (i) imply that $\bar{M}_{i}=K_{i},\left\{M_{i}: i \in I\right\}$ is a dense subset of $X(\mathscr{Q})$ and that $M_{i} \neq M_{i}$ ' whenever $i \neq i^{\prime}$. Similar facts are true about the $N_{j}$ 's. The notion of irredundancy used in 4.3 (ii) is so strong we can prove the following.

(1) Every $M_{i}$ (and similarly $N_{j}$ ) is an isolated point of $X(2)$.

$B y$ the irredundancy condition on the $B_{i}$ 's and the regularity of the ideals, $\left\{M_{i}: \quad i \in I, i \neq i_{0}\right\}$ is not dense in $X(\mathscr{U})$. Thus, there is a clopen subset $N$ such that $M_{i_{0}} \in N$ and $N$ is disjoint from $\left\{M_{i}: i \in I, i \neq i_{0}\right\}$. Since $X(\mathscr{V})$ is Hausdorff and $\left\{M_{i}: i \in I\right\}$ is dense, $\left\{M_{i}\right\}=N$; thus, $M_{i_{0}}$ is isolated.

Since isolated points of $X(\mathscr{U})$ correspond to maximal principal ideals of $Z(\mathscr{U})$, it follows from (1) that, for every $i \in I, M_{i}$ is the ideal generated by the dual atom $a_{i}$ of $Z(\mathscr{U})$. Since $\bigcap_{i \in I} \bar{M}_{i}=\{0\}$, it follows that $\left\{a_{i}: i \in I\right\}$ is the set of all dual atoms of $Z(\mathscr{U})$. Similarly, for each $j \in J, N_{j}$ is the principal ideal generated by a dual atom $b_{j}$ of $Z(\mathscr{U})$ and $\left\{b_{j}: j \in J\right\}$ is the set of all dual atoms of $Z(2)$. Since $a_{i} \neq a_{i}^{\prime}$ for $i \neq i^{\prime}$ and $b_{j} \neq b_{j}^{\prime}$ for $j \neq j^{\prime}$, it follows that 
$|I|=|J|$ and there is a one-to-one function $f$ of $I$ onto $J$ such that $N_{f(i)}=M_{i}$ for all $i \in I$. Since $\mathcal{Q}$ is regular $K_{i}=L_{f(i)}$ and so $\mathbb{B}_{i} \cong \mathcal{S}_{f(i)}$ for all $i \in I$ as desired.

Corollary 4.5. Every $L C A_{\alpha}$ (and in particular, every $C A_{\alpha}$ if $a<\omega$ ) bas the unique subdirect decomposition property 4.3 (ii).

Along with the uniqueness problem for subdirect decompositions there is also the question of the existence of subdirect decompositions of 2 into subdirectly indecomposable $\mathrm{CA}_{\alpha}$ 's which is irredundant in the strong sense of 4.3 (ii). The proof of 4.4 shows that if 20 is regular the existence of such a subdirect decomposition implies $Z(2)$ is atomic. It is easily seen that this condition is not sufficient. In fact, for $\mathrm{CA}_{0}$ 's, i.e. BA's, such an irredundant decomposition exists for $\mathcal{Q}$ if and only if $\mathcal{A}$ is finite.

As mentioned above 4.3 (i) holds for every $\mathrm{CA}_{\alpha}$ under the additional assumption that $|I|,|J|<\omega$. The following examples show this is not true for arbitrary $I$ and $J$.

Proposition 4.6. There exist a $C A_{\alpha}$ \&l and two systems of ideals $\left\langle K_{i}\right.$ : $i \in I\rangle$ and $\left\langle L_{j}: i \in I\right\rangle$ satisfying (1)-(3) in $4.3(i)$ such that, for some $j \in I$, $L_{j} \notin\left\{K_{i}: i \in I\right\}$. Thus, the unique subdirect decomposition property 4.3 (i) fails.

Proof. Let $B$ be a subdirectly indecomposable $C A_{\alpha}$ that is not simple and let $X={ }^{\omega} 2$ be the Stone space of the free BA on $\omega$ generators. The algebra $\mathcal{O}$ we want is $\mathcal{A}=\Gamma(X, \mathcal{S})$ where $\mathcal{S}$ is the trivial $\mathbb{B}$-sheaf over $X$. Results involved in the proof of the duality theorem (Theorem 1.2) allow us to write down subdirect representations of $\mathcal{U}$. Namely, for $x \in X$, let $M_{x}=\left\{\sigma \in Z(\mathcal{U}): \sigma(x)=0_{x}\right\}$; the system of ideals $\left\langle\bar{M}_{x}: x \in X\right\rangle$ satisfies (1)-(3) above. For the system of ideals $\left\langle K_{i}: i \in I\right\rangle$ let $I=X$ and $K_{x}=\bar{M}_{x}$ for $x \in X$. Fix $j \in I$ and define the ideal $L_{i}$ for $i \neq j$ by $L_{i}=\bar{M}_{i}$; let $L_{j}=\{\sigma \in A ; \sigma(j) \in N\}$ where $N$ is some maximal ideal of the stalk $\mathcal{S}_{j} \cong \mathbb{B}$. Clearly $L_{j}$ is an ideal such that $L_{j} \supset M_{j}$. For all $i \in I, Q \mathrm{U} / L_{i}$ is subdirectly indecomposable and, since $X$ is Hausdorff, $L_{x} \varsubsetneqq L_{y}$ for $x, y \in X, x \neq y$. Before proving that $\bigcap_{i \in I} L_{i}=\{0\}$, observe the following consequence of the partition property.

(4) For any $\sigma \in A$ there exists a partition $\left\{N_{i}: i<n\right\}$ of $X$ into a finite number of clopen subsets such that $\mathrm{pr}_{1} \circ \sigma$ is constant on each $N_{i}\left(\mathrm{pr}_{1}\right.$ is the natural projection of $X \times \mathbb{B}$ onto $B$ ).

Now suppose $0 \neq \sigma \in A$. By (4) there exist a clopen subset $N$ of $X$ such that $\sigma(x) \neq 00_{x}$ for ali $x \in N$. Since $\{j\}$ is not open there is an $x \in N, x \neq j$. Since $\sigma(x) \neq 0_{x}, \sigma \notin \bar{M}_{x}=L_{x}$. Thus $\bigcap_{x \in X} L_{x}=\{0\}$. The two systems of ideals $\left\langle K_{x}: x \in X\right\rangle$ and $\left\langle L_{x}: x \in X\right\rangle$ in 20 satisfy 4.6 .

Instead of using, for $X$, the Cantor space ${ }^{\omega} 2$ we could have used any non- 
discrete Boolean space; we must only find some $j \in X$ such that $\{j\}$ is not open. A disadvantage of 4.6 is that the construction will not give an $\mathrm{LCA}_{\alpha}$ थ since directly indecomposable $\mathrm{LCA}_{\alpha}$ 's are simple. The next result can yield LCA 's and indicates how $|I|$ and $|J|$ may differ.

Proposition 4.7. There exist a $C A_{\alpha}$ Ul (which can be chosen an LCA $A_{\alpha}$ if desired $)$ and two systems $\left\langle K_{i}: i \in I\right\rangle$ and $\left\langle L_{j}: j \in J\right\rangle$ of ideals of $\mathcal{O l}$ satis. fying conditions (1)-(3) in 4.3 (i) but for which $|I| \neq|J|$.

Proof. Let $B$ be a subdirectly indecomposable $\mathrm{CA}_{a}$ and $X={ }^{\omega} 2$. Choose $\mathcal{U}=\Gamma(X, \mathcal{S})$ where $\mathcal{S}$ is the trivial $B$-sheaf over $X$. Note $\mathcal{U}$ is an $\mathrm{LCA}_{\alpha}$ if $B$ is. The system of ideals $\left\langle\bar{M}_{x}: x \in X\right\rangle$ satisfies (1)-(3). $X$ has a countable dense subset $J$. The system of ideals $\left\langle\bar{M}_{x}: x \in J\right\rangle$ of 2 also satisfies (1)-(3); (1) is a consequence of $J$ being dense and property (4) from the proof of 4.6. The result follows since $|X|>|J|$.

\section{BIBLIOGRAPHY}

1. S. Comer, Representations by algebras of sections over Boolean spaces, Pacific J. Math. 38 (1971), 29-38.

2. W. Hanf, On some fundamental problem concerning is omorphism of Boolean algebras, Math Scand. 5 (1957), 205-217. MR $21 \nRightarrow 7167$.

3. L. Henkin, D. Monk and A. Tarski, Cylindric algebras, I, North-Holland, Amsterdam, 1971 .

4. L. Henkin and A. Tarski, Cylindric algebras, Proc. Sympos. Pure Math., vol. 2, Amer. Math. Soc., Providence, R. I., 1961, Pp. 83-113. MR 23 \#A1564.

5. B. Jónsson, On isomorphism types of groups and other algebraic systems, Math. Scand. 5 (1957), 224-229. MR 29 \#7169.

6. R. S. Pierce, Modules over commutative regular rings, Mem. Amer. Math. Soc. No. 70 (1967). MR $36 \# 151$. 37203

DEPARTMENT OF MATHEMATICS, VANDERBILT UNIVERSITY, NASHVILLE, TENNESSEE 